www.nature.com/pj

\title{
The rheological behavior and thermal conductivity of melt-compounded polycarbonate/vapor-grown carbon fiber composites
}

\author{
Jittiwat Nithikarnjanatharn, Hisai Ueda, Shuichi Tanoue, Hideyuki Uematsu and Yoshiyuki Iemoto
}

The effect of polycarbonate (PC) matrix viscosity on the thermal conductivity of a PC/vapor-grown carbon fiber (VGCF) composite was investigated in this study in terms of the rheological properties of the PC/VGCF using two types of VGCF. Two types of VGCF, which have different aspect ratios (VGCF-h has an aspect ratio of 40 , whereas VGCF-s has an aspect ratio of 100), were added to two types of PC with different viscosities. The storage modulus $\left(G^{\prime}\right)$ and loss modulus $\left(G^{\prime}\right)$ of the PC slightly increased and thermal conductivity gradually increased with the content of VGCF-h. By adding VGCF-s to low-viscosity PC, rheological properties originating in the network structure were observed. Thermal conductivity of low-viscosity PC drastically increased with the content of VGCF-s. By analyzing the length of VGCF in each composite, we found that the length of VGCF decreased with mixing. It was also easy to shorten VGCF in high-viscosity PC. We clarified that the thermal conductivity of PC/VGCF could be controlled with the viscosity of the polymer matrix because the spread of the network structure of VGCF and/or the breaking of VGCF depended on the viscosity of the polymer matrix.

Polymer Journal (2012) 44, 427-432; doi:10.1038/pj.2011.149; published online 25 January 2012

Keywords: length of VGCF; rheological properties; thermal conductivity; VGCF composites

\section{INTRODUCTION}

Many technological fields, including electronics, vehicles and the life sciences, require materials that are versatile and functional in various ways. One of the material classes that fit these requirements is polymer materials because they are highly processable. The development of polymeric materials is indispensable for providing novel functionalities to products and is roughly divided into two methods: molecular and material design. The study of material design by adding an inorganic material with a high functionality has been thoroughly investigated. Titanium dioxide as photoactive catalytic, electronically conductive silver particles, PZT (lead zirconate titanate) with piezoelectric properties and clay as toughening agents have been incorporated into and studied in polymeric materials. ${ }^{1-4}$ The dispersion of inorganic material is important to control both thermal and optical properties. In these reports, dispersion of the inorganic material could be controlled with surfactant agents and surface treatment. Recently, carbon fiber has been expected to be a good filler for polymer composites due to the ability to control its mechanical and electrical properties. ${ }^{5-7}$ It is interesting that epoxy resin is used as a binder of carbon fiber. This is known as a carbon fiber-reinforced polymer. As the main material is carbon fiber, the physical properties of the material assume those of the carbon fiber. The use of carbon nanotubes (CNTs) as an additive agent to the polymeric material in fibrous carbon material is a current focus, and is expected to create a very electrically and thermally conductive material.

Carbon nanotubes are cylindrical versions of mono- or multi-layer graphene sheets. The diameter of a CNT depends on the number of layers and can be controlled by processing methods. ${ }^{8-11}$ As previously shown in other reports, the diameter and length of CNTs are often on the order of $10 \mathrm{~nm}$ and $100 \mu \mathrm{m}$, respectively. At present, vapor-grown carbon fiber (VGCF) is a new class of carbon fiber that is distinct from other types of carbon fibers because of its production method. ${ }^{12}$ VGCF is a highly crystalline carbon nanofiber synthesized by a gas-phase method. The diameters and lengths of VGCF often are $100-200 \mathrm{~nm}$ and below $100 \mu \mathrm{m}$, respectively. There is also a difference in fiber structure between VGCF and CNTs; VGCF has a relatively linear fiber structure and a larger diameter than do CNTs and consists of mostly curved tubes. ${ }^{13}$ VGCF has been an industrial focus because the production efficiency of VGCF is higher than that of the conventional CNT processing method. The VGCF structure exhibits excellent electrical conductivity, thermal conductivity, strength, lubricity and resiliency for fibers with a high degree of graphitization. ${ }^{14-15}$ One of the most important challenges in improving the properties of composites based on VGCF is obtaining a uniform dispersion of VGCF

Fiber Amenity Engineering, Graduate School of Engineering, University of Fukui, Bunkyo, Fukui, Japan

Correspondence: Dr S Tanoue, Graduate School of Engineering, University of Fukui, 3-9-1 Bunkyo, Fukui 910-8507, Japan. E-mail: tanoue@matse.u-fukui.ac.jp

Received 24 August 2011; revised 29 November 2011; accepted 1 December 2011; published online 25 January 2012 
within the polymer matrix. ${ }^{16,17}$ VGCF-reinforced polymer composites have potential applications as conducting polymeric materials, particularly to promote stiffness and thermal properties, and are classified as a new generation of polymer composites and alternative composite materials for aerospace, automotive and structural applications. Koyama et al. ${ }^{18}$ have reported that the tensile properties of polypropylene/VGCF composites and monofilaments were improved by melt compounding using a twin screw extruder and fiber spinning operation even if the VGCF content is very small ( $1 \mathrm{wt} \%$ in this study). Koyama et al. ${ }^{19}$ studied the effects of matrix viscosity and screw rotation speed on the properties of polyamide 6/VGCF composites by melt compounding. They found that matrix viscosity influences the tensile properties of polyamide 6/VGCF composites when VGCF has a high aspect ratio and does not influence the tensile properties of polyamide 6/VGCF composites when VGCF has a low aspect ratio. However, because this result needs to consider the effect of VGCF on crystallization of the polymer, the relationship between physical properties, including tensile properties and the dispersion state of VGCF, is not immediately clear. Takase et al. ${ }^{20,21}$ studied the dispersion state of VGCF in polycarbonate (PC), which is an amorphous polymer, by melt compounding. However, they did not focus on the effect of the melt viscosity of the polymer matrix on the dispersion state of VGCF.

Fiber suspensions generally have remarkably higher viscosity and elasticity than spherical particle suspensions at the same volume concentration. For example, Potschke et al. ${ }^{22}$ investigated the rheological properties of PC/CNT composites. The increase in viscosity associated with the addition of CNTs was much higher than that of carbon black. The rheological behavior for CNTdispersed suspension was also studied by Kinloch et al. ${ }^{23}$ They have examined the network structure of CNTs to determine the elasticity components measured in oscillatory shear. On the basis of these studies, it can be concluded that viscosity of the matrix polymer increases significantly by adding rigid, rod-like material because of the increase in the interparticle interaction compared with the polymer/particle system. In a polymer suspension contained in a pulp fiber system, Mason ${ }^{24}$ proposed the equation for the critical concentration to form a network structure of pulp fiber. The same researchers have also obtained the mathematical relationship between the modulus and concentration in a pulp fiber suspension system. ${ }^{25-29}$ Tatsumi ${ }^{30}$ has reported that the elastic modulus and the concentration of the pulp fiber are related by a power law. On the basis of the polymer gel solution system, Tatsumi has also reported that a power law could be theoretically expressed in the microfiber suspension system. ${ }^{31}$ In other words, it is suggested that the dispersion state of the fiber can be indirectly estimated with melt rheological properties. Therefore, rheological properties are useful information in the investigation of the effect of the dispersion state of VGCF on the thermal and electrical properties. However, the relationship between rheological properties and thermal properties has not been intensely studied. In particular, the effect of viscosity of the matrix polymer on the dispersion of VGCF has not been investigated in sufficient detail.

In this study, we investigated the effect of the viscosity of the matrix polymer on the thermal conductivity of VGCF composites from the viewpoint of the rheological properties of PC/VGCF.

\section{EXPERIMENTAL PROCEDURE}

\section{Materials}

Two types of PC (K1300 and 1225L, provided from Teijin Chemical, Tokyo, Japan) with different melt volume flow rates were used as matrix polymers. Table 1 shows the characteristic properties of PC.
Table 1 Characteristics of polycarbonate (PC)

\begin{tabular}{ccccc}
\hline Code & Grade & $M_{n}\left(\mathrm{gmol}^{-1}\right)$ & $M_{w}\left(\mathrm{gmol}^{-1}\right)$ & MVR(cm \\
\hline PC-H & K1300 & 22100 & 57800 & 2.5 \\
PC-L & $1225 \mathrm{~L}$ & 16200 & 36400 & 18 \\
\hline
\end{tabular}

Table 2 Characteristics of vapor-grown carbon fiber (VGCF)

\begin{tabular}{lccccc}
\hline Code & Grade & $\begin{array}{c}\text { Diameter } \\
(\mathrm{nm})^{\mathrm{a}}\end{array}$ & $\begin{array}{c}\text { Length } \\
(\mu \mathrm{m})^{\mathrm{a}}\end{array}$ & $\begin{array}{c}\text { Aspect } \\
\text { ratio }\end{array}$ & $\begin{array}{c}\text { Thermal } \\
\text { conductivity } \\
(W /(m \cdot K))\end{array}$ \\
\hline VGCF-S & VGCF-S & 100 & 10 & 100 & 1200 \\
VGCF-h & VGCF-H & 150 & 6 & 40 & 1200 \\
\hline
\end{tabular}

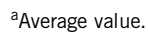

We labeled K1300 as 'PC-H' and 1225L as 'PC-L.' We selected PC, which is a typical amorphous polymer, as the matrix polymer to discuss the dispersion of VGCF without the effect of a crystalline component of the polymer. Two types of VGCF (VGCF-S and VGCF-H) made by Showa Denko KK (Japan) were added to PC. Table 2 shows the characteristic properties of VGCF. We labeled VGCF-S as 'VGCF-s' and VGCF-H as 'VGCF-h. The VGCF content ranged from 0 to 6.5 wt $\%$. Both PC and VGCF were dried at $80^{\circ} \mathrm{C}$ in a decompression state for $24 \mathrm{~h}$. The composite was prepared using a twin screw extruder (KRC-S1 kneader, Kurimoto, Osaka, Japan) at $260^{\circ} \mathrm{C}$. The twin screw rotation speed was 150 r.p.m. The mixed samples were pressed for rheological measurement at $260^{\circ} \mathrm{C}$ and $10 \mathrm{MPa}$ into a disk with a thickness of $0.5 \mathrm{~mm}$ and diameter of $25 \mathrm{~mm}$. For thermal conductivity measurement, the mixed samples were molded under the same conditions into a sheet with a thickness of $1 \mathrm{~mm}$, width of $130 \mathrm{~mm}$ and length of $150 \mathrm{~mm}$.

\section{Measurement procedure}

Dynamic rheological measurements of PC/VGCF composites were carried out at $260{ }^{\circ} \mathrm{C}$ with a strain-controlled rheometer (ARES, TA Instruments, New Castle, DE, USA) using parallel plates as a fixture. Parallel plates with a $25 \mathrm{~mm}$ diameter were used for frequency $(\omega)$ sweeps. The gap between the parallel plates was $\sim 0.4 \mathrm{~mm}$. The $\omega$ dependencies of the storage and loss moduli, $G^{\prime}$ and $G^{\prime \prime}$, were measured in the frequency range from 0.1 to $100 \mathrm{rad} \mathrm{s}^{-1}$. The strain ranged from 0.01 to 0.2 in the linear viscoelastic condition for $\mathrm{PC} /$ VGCF systems. The moduli $G^{\prime}$ and $G^{\prime \prime}$ were measured without thermal degradation in a frequency sweep test.

The thermal conductivity of the PC/VGCF composite was measured using QTM-500 (Kyoto Electronics Manufacturing, Kyoto, Japan) at room temperature. The sensor shape was a rectangle with a length of $95 \mathrm{~mm}$ and a width of $15 \mathrm{~mm}$. The thermal conductivities of the samples were quickly evaluated according to the following equation by using a hot wire method ${ }^{32}$

$$
\lambda=\frac{q \ln \left(t_{2} / t_{1}\right)}{4 \pi\left(T_{2}-T_{1}\right)}
$$

where $\lambda$ refers to the thermal conductivity of the sample and $q$ is the generated heat flux per unit length. $T_{1}$ and $T_{2}$ are the temperatures at times $t_{1}$ and $t_{2}$, respectively. The thermal conductivity of each sample was measured on a flat surface without the effects of thickness irregularities on thermal conductivity. In addition, we used reference 
plates and software for measuring thermal conductivity of sheet-type samples.

Scanning electron microscopy (SEM) was performed using S-2600HS (Hitachi, Tokyo, Japan) to investigate the length of the VGCF. According to previous reports, ${ }^{33,34}$ SEM was used to measure the length of VGCF. After stirring the dilute PC/VGCF solution dissolved in tetrahydrofuran, thin films of PC/VGCF were prepared using a rotational coater at various rotating speeds. The PC component in the PC/VGCF film was carbonized at $500{ }^{\circ} \mathrm{C}$ for $20 \mathrm{~min}$ to capture a clear image. VGCF lengths were determined from SEM images, except for the case of inter-tangling VGCF, for which the length was determined by software (Luzex AP, Nireco Corporation, Hachioji, Japan). In all, 200 segments of VGCF were analyzed because the average length of the VGCF did not change when more segments were analyzed.

\section{RESULTS AND DISCUSSION}

\section{Rheological behavior}

Figure 1 shows the frequency $(\omega)$ dependence of the storage modulus $G^{\prime}$ and the loss modulus $G^{\prime \prime}$ of the PC-H/VGCF-h composite at $260^{\circ} \mathrm{C}$. In the low- $\omega$ region $\left(<1.0 \mathrm{rad} \mathrm{s}^{-1}\right)$, the slope of $G^{\prime}$ and $G^{\prime \prime}$ for
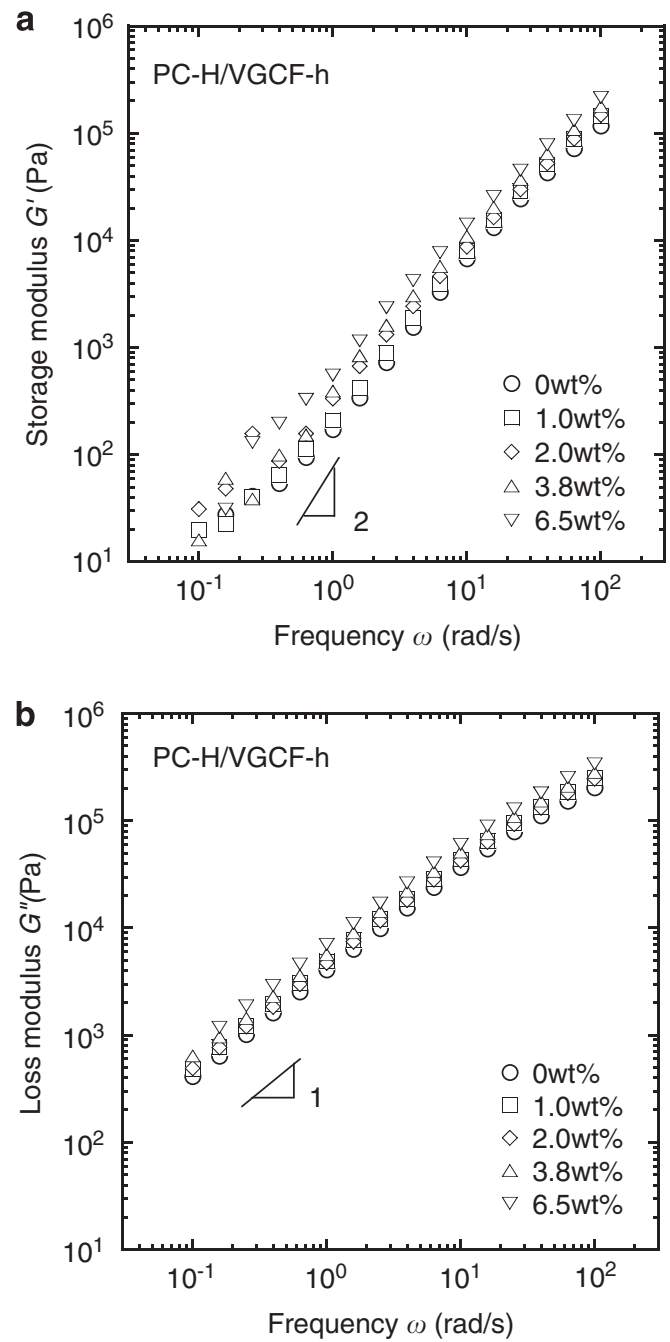

Figure 1 Frequency $(\omega)$ dependence of the linear viscoelastic moduli: (a) storage modulus $G^{\prime}$ and (b) loss modulus $G^{\prime \prime}$ for PC-H/VGCF-h system at $260^{\circ} \mathrm{C}$. PC, polycarbonate; VGCF, vapor-grown carbon fiber. the PC-H matrix was 2 and 1, respectively. In other words, terminal relaxation behavior was observed. However, the $G^{\prime}$ and $G^{\prime \prime}$ values of PC-H monotonically increased with the content of VGCF-h. The slopes of $G^{\prime}$ and $G^{\prime \prime}$ for PC-H/VGCF-h were almost independent of the content of VGCF-h. We found that the effect of VGCF-h on rheological properties of $\mathrm{PC}-\mathrm{H}$ was small. This result implies that VGCF-h may disperse well in PC-H. In Figure 2, $G^{\prime}$ and $G^{\prime \prime}$ are shown in the PC-H/VGCF-s system. The $G^{\prime}$ and $G^{\prime \prime}$ of PC-H increased monotonically in the high- $\omega$ region $\left(>10 \mathrm{rads}^{-1}\right)$ with increasing VGCF-s content. However, in the low- $\omega$ region $\left(<1.0 \mathrm{rad} \mathrm{s}^{-1}\right)$, the $G^{\prime}$ and $G^{\prime \prime}$ of PC-H rapidly increased and the slopes of $G^{\prime}$ and $G^{\prime \prime}$ gradually decreased with VGCF-s content above $2.0 \mathrm{wt} \%$. In other words, the long-time relaxation behavior was observed and the relaxation component slightly increased in the region of $>2.0 \mathrm{wt} \%$ in the PC-H/VGCF-s system. This result implies that the dispersion state of VGCF-s in PC-H would change in the region of $>2.0 \mathrm{wt} \%$. Figure 3 shows the $G^{\prime}$ and $G^{\prime \prime}$ values as a function of $\omega$ in the PC-L/ VGCF-s system. As observed in Figure 3, terminal relaxation behavior was observed in the PC-L matrix, as in the PC-H matrix. The complex viscosity $\eta^{*}\left(\eta^{*}=\left[\left(G^{\prime \prime} / \omega\right)^{2}+\left(G^{\prime} / \omega\right)^{2}\right]^{1 / 2}\right)$ of PC-H and PC-L was almost independent of the frequency $\omega$ in our measurement region.
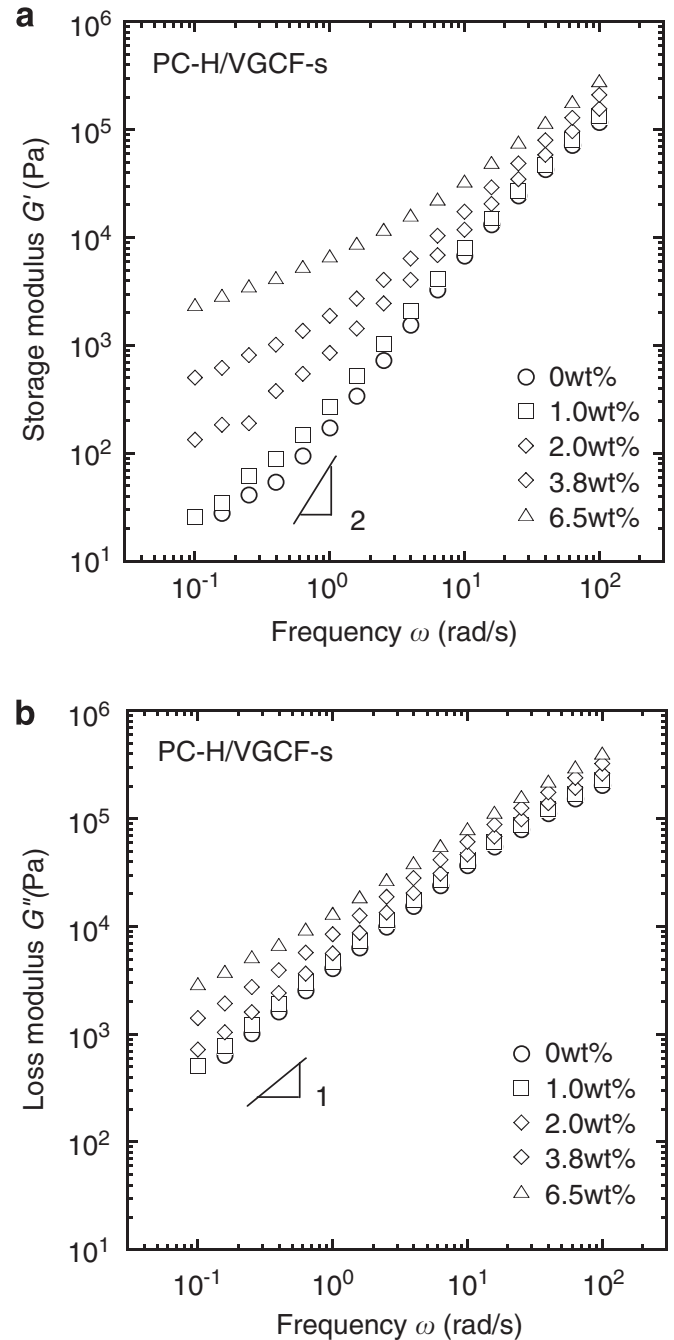

Figure 2 Frequency $(\omega)$ dependence of the linear viscoelastic moduli: (a) storage modulus $G^{\prime}$ and (b) loss modulus $G^{\prime \prime}$ for PC-H/NGCF-s system at $260^{\circ} \mathrm{C}$. PC, polycarbonate; VGCF, vapor-grown carbon fiber. 

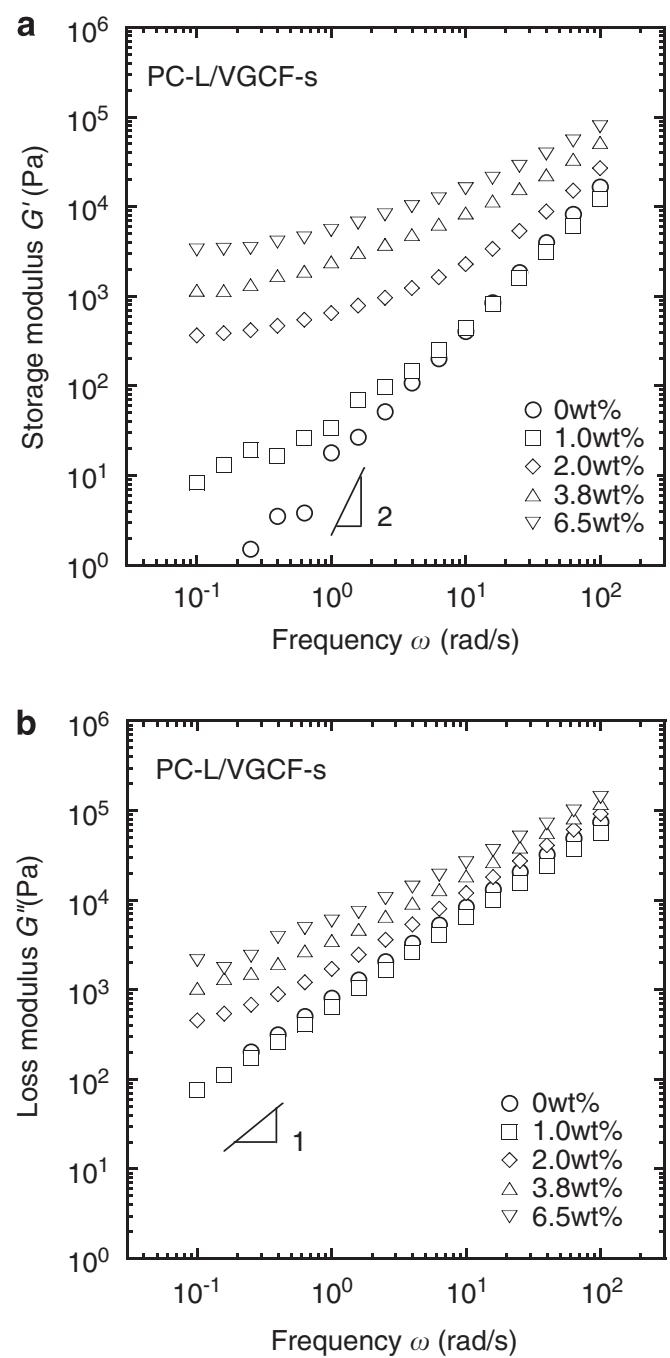

Figure 3 Frequency $(\omega)$ dependence of the linear viscoelastic moduli: (a) storage modulus $G^{\prime}$ and (b) loss modulus $G^{\prime \prime}$ for PC-L/VGCF-s system at $260^{\circ} \mathrm{C}$. PC, polycarbonate; VGCF, vapor-grown carbon fiber.

We found that $\eta^{*}$ of PC-H and PC-L were 4000 and $700 \mathrm{Pas}$, respectively, and that the $G^{\prime}$ and $G^{\prime \prime}$ of PC-L/VGCF-s dramatically increased above $2.0 \mathrm{wt} \%$. In the low- $\omega$ region $\left(<0.4 \mathrm{rad} \mathrm{s}^{-1}\right)$, the slope of $G^{\prime}$ for PC-L/VGCF-s was almost flat and the magnitude of $G^{\prime}$ was only slightly affected by the content of VGCF-s. In other words, the second plateau region was observed above $2.0 \mathrm{wt} \%$ in the PC-L/VGCF-s system. This result implies that the structure with low mobility would be formed in PC-L by adding VGCF-s.

The elastic component of the polymer can be increased by adding rigid particles, ${ }^{1-4}$ and it is well known that the elasticity of fiber suspensions (for example, PC/multiwall CNT composites ${ }^{22}$ or aqueous multi-walled CNT dilute suspensions ${ }^{23}$ ) drastically increases with their concentration. ${ }^{22-23}$ These results correspond to our obtained results. In addition, some researchers have reported that the plateau modulus $G^{\prime}$ in the low- $\omega$ region reflected the properties of the fiber network structure. ${ }^{24-30}$ According to these reports, a power law correlation was found between the plateau modulus $G^{\prime}$ and the volume concentration $c_{v}$ namely, $G^{\prime}=k c_{v}{ }^{a}$ where $k$ and $a$ are parameters that are independent of $c_{v}$. Tatsumi et al. ${ }^{29,30}$ have also explained that the $k$ is the index of fiber flexibility, which depends
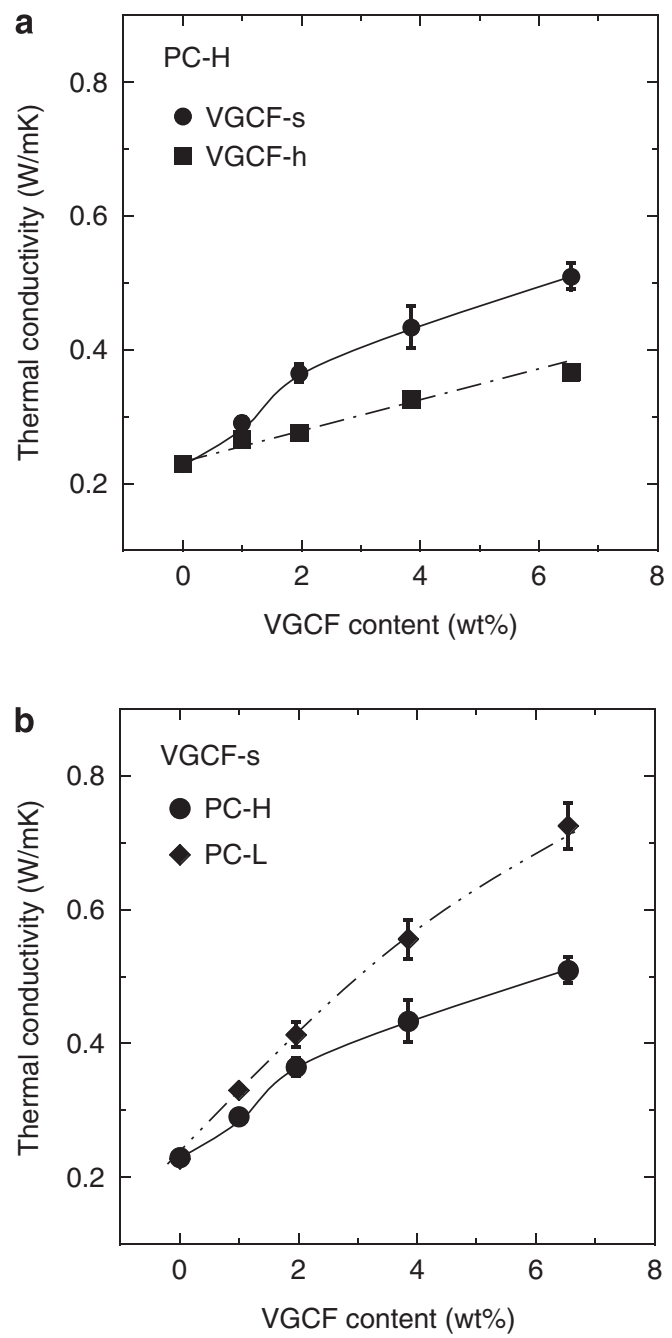

Figure 4 Thermal conductivity plotted against the content of VGCF for the (a) PC-H/VGCF system and (b) PC/NGCF-s system at room temperature. PC, polycarbonate; VGCF, vapor-grown carbon fiber.

on Young's modulus and the aspect ratio of the fiber. The result of the investigation of the plateau modulus and the content of VGCF in Figure 3 was that $\log G^{\prime}$ was proportional to $\log c_{v}$ of VGCF-s in the PC-L/VGCF-s system. This result implies that the network structure of VGCF-s is formed in the PC-L matrix. Notably, our data indicate that the network structure of VGCF-s cannot be expressly formed in the PC$\mathrm{H}$ matrix. This result leads to our presumption that the network structure of VGCF is locally formed or that the coarse network structure of VGCF is formed in the PC-H matrix. In other words, it is not easy to form a network structure of VGCF in a high-viscosity matrix.

\section{Thermal conductivity}

Figure 4a shows thermal conductivity as a function of VGCF content for the PC-H/VGCF system. The thermal conductivity of PC-H/ VGCF-h slightly increased with increasing VGCF-h content, and the thermal conductivity of $\mathrm{PC}-\mathrm{H}$ was observed to strongly increase by adding VGCF-s above $2.0 \mathrm{wt} \%$ compared with that in the PC-H/ VGCF-h system. In Figure $4 \mathrm{~b}$, the thermal conductivities of PC-H and PC-L were plotted as a function of VGCF-s content. The thermal conductivity of each PC was almost the same below $2.0 \mathrm{wt} \%$; however, 
the thermal conductivity of PC-L drastically increased above $2.0 \mathrm{wt} \%$ compared with that of $\mathrm{PC}-\mathrm{H}$.

It is predicted that the thermal conductivity of PC/VGCF composites depends on the degree of VGCF connections because the thermal conductivity of VGCF is much higher than that of PC. From the result shown in Figure 3, we suggest that the thermal conductivity of PC-L drastically increased above $2 \mathrm{wt} \%$ because the network structure of VGCF-s would be formed in the PC-L matrix. As the network structure of VGCF-h would not be sufficiently formed in $\mathrm{PC}-\mathrm{H}$, the thermal conductivity of PC-H/VGCF-h was lower than that of PC-H/ VGCF-s. These observations indicate that the effect of VGCF on the thermal conductivity of PC may be interpreted from the results of the rheological behavior of the composite. A further important point is that thermal conductivity and rheological behavior depend on the viscosity of the PC matrix despite the fact that the same amount of VGCF is added to both samples. We investigated the morphology of PC-H/VGCF-s and PC-L/VGCF-s with SEM. ${ }^{35}$ The flocculating of VGCF was only minimally observed in each composite. However, it is difficult to discuss the dispersion state of VGCF because a clear network structure of VGCF could not be observed. To investigate the effect of viscosity of the PC matrix on the formation of network of VGCF, we focused on the length of VGCF in various PCs.

\section{Length of VGCF in the composites}

The length of VGCF was investigated by evaluating the disaggregated VGCF, with reference to previous reports. ${ }^{33,34}$ After stirring neat VGCF-h and VGCF-s in dilute PC/tetrahydrofuran solution, a very thin film cast by rotary coater was observed with SEM. The image and
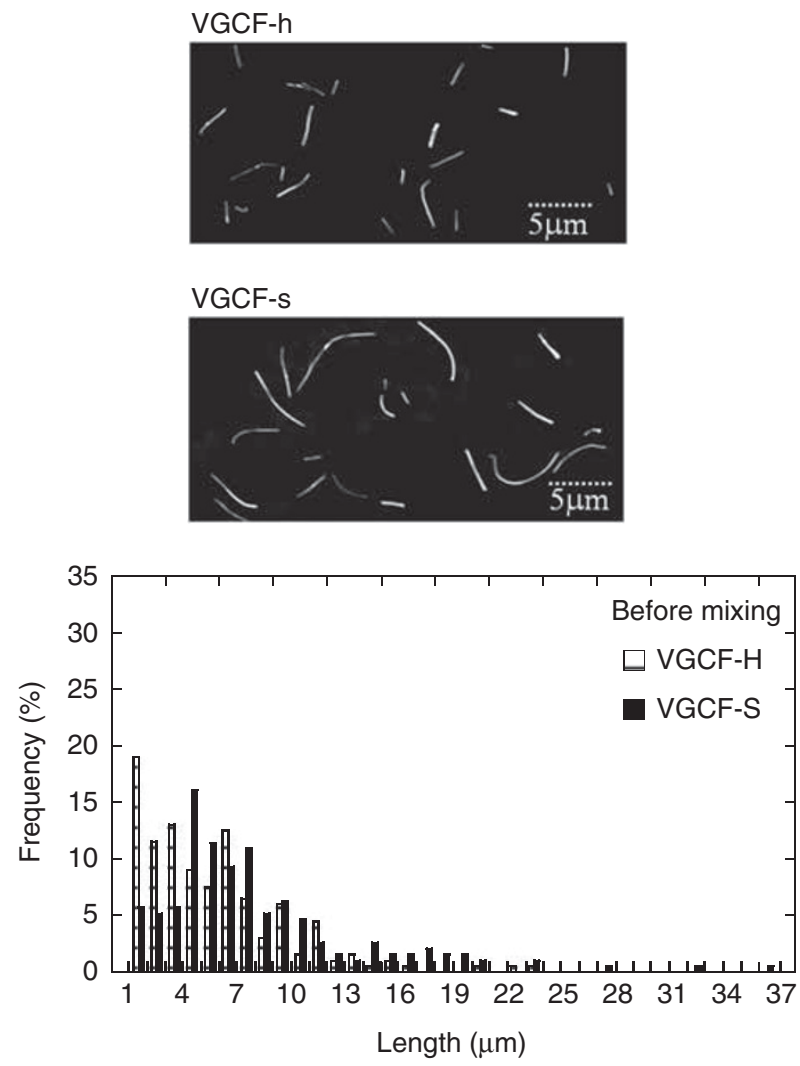

Figure 5 Length distribution and the pictures for VGCF-S and VGCF-h before mixing. VGCF, vapor-grown carbon fiber. the length distribution of VGCF-h and VGCF-s before mixing are shown in Figure 5. The number of VGCF segments analyzed was $\sim 200$ because the average length of VGCF was independent of the number of VGCF segments analyzed above 150 in each case. The class interval was $1 \mu \mathrm{m}$, and each class bar was plotted as $1 \mu \mathrm{m}$. As observed in the images and figure, a longer VGCF was confirmed in VGCF-s than in VGCF-h. The important point is the shape of the length distribution. The shapes of VGCF-h and VGCF-s distributions resemble single and bimodal distributions, respectively, in Figure 5. The length distribution of VGCF-h and VGCF-s after mixing with PC-H at a VGCF content of $6.5 \mathrm{wt} \%$ is shown in Figure $6 \mathrm{a}$. We found that the average length of VGCF-s and VGCF-h decreased by $\sim 40 \%$ after mixing with PC-H. However, a broader distribution in the PC-H/ VGCF-s system than in the PC-H/VGCF-h system was observed. The averages of VGCF-s and VGCF-h were 4.9 and $3.3 \mu \mathrm{m}$, respectively. The ratio of breaking for VGCF-s was more than that of VGCF-h. Figure $6 \mathrm{~b}$ shows the result of PC-L/VGCF-s and PC-H/VGCF-s at a VGCF content of $6.5 \mathrm{wt} \%$. It was also found that the length of VGCF-s decreased with mixing. However, the shapes of the length distribution of VGCF-s in PC-H and PC-L resemble single and bimodal distributions, respectively. The average length of VGCF-s in PC-H and PC-L were 4.9 and $5.9 \mu \mathrm{m}$, respectively. This result implies that it is easy to break VGCF in the kneading process in a high viscosity matrix.

The diameter of VGCF-s was larger than that of VGCF-h at the same VGCF content because the volume per fiber of VGCF-s was $25 \%$ lower than that of VGCF-h, as shown in Table 2 before mixing. The surface area per fiber of VGCF-s was also $\sim 10 \%$ larger than that of VGCF-h. However, the number of VGCF-s also increased with mixing
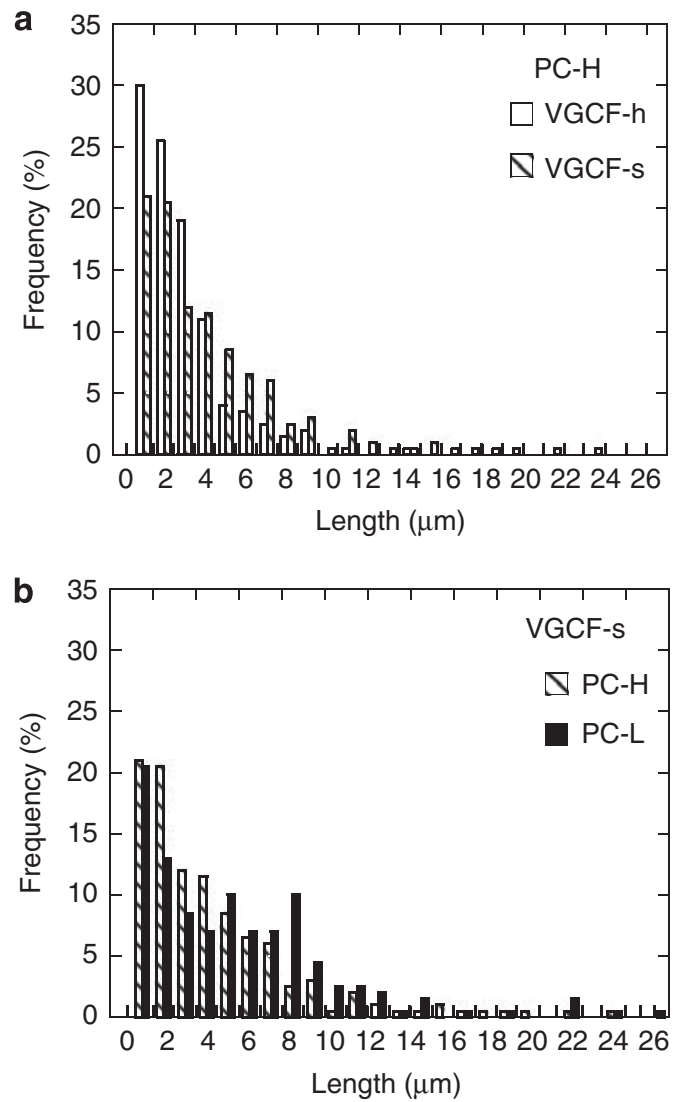

Figure 6 Length distribution of (a) VGCF-h and VGCF-S in PC-H and (b) VGCF-s in PC-H and PC-L after mixing at VGCF content of 6.5 wt\%. $\mathrm{PC}$, polycarbonate; VGCF, vapor-grown carbon fiber. 
because the ratio of breaking for VGCF-s was more than that for VGCF-h. The total contact point between VGCF-s was smaller than that of VGCF-h after mixing. Therefore, it is very interesting that the thermal conductivity of the PC-H/VGCF-s system is so different from that of the PC-L/VGCF-s system, although the average length of VGCF-s in the PC-H/VGCF-s system is a little larger (the difference is $\sim 1 \mu \mathrm{m}$ ) compared with that in PC-L/VGCF-s. In this study, we clarified that viscosity of the polymer matrix is an important factor to assure that the VGCF will have sufficient effects on the properties of the PC/VGCF composite. Therefore, these results suggest that the dispersion of VGCF may be controlled with temperature because viscosity of PC depends on the temperature. The effect of temperature on the dispersion of VGCF will be the subject of future work.

\section{CONCLUSION}

We investigated the effects of PC matrix viscosity on the thermal conductivity of a PC/VGCF composite from the viewpoint of the rheological properties. Two types of VGCF with aspect ratios of 40 and 100 were used in this study. The content of VGCF ranged from 0 to $6.5 \mathrm{wt} \%$. The rheological behavior results from the network structure of VGCF were observed in the low-viscosity PC system that contained VGCF with a high aspect ratio because a power law correlation was found between the plateau modulus and the volume concentration. In the high-viscosity PC system, the rheological behavior originating from the network structure of VGCF was not shown. The thermal conductivity of low-viscosity PC drastically increased by adding VGCF with a high aspect ratio, in contrast to the result from highviscosity PC. From the analysis of the VGCF length in each composite, it was clearly found that VGCF length decreased with mixing. However, the degree of VGCF length decrease in the high-viscosity PC was higher. Therefore, we concluded that the thermal conductivity of polymer/ VGCF composites could be controlled with the viscosity of the polymer because the length of the VGCF changed as a function of the viscosity of the matrix.

\section{CONFLICT OF INTEREST}

The authors declare no conflict of interest.

\section{ACKNOWLEDGEMENTS}

We thank Mr Yuki Kurome, a student at the University of Fukui, Japan, for measuring the length distributions of VGCF in PC/VGCF composites and Teijin Chemicals, Japan, for supplying the PC resin.

1 Leyland, N. S., Evans, J. R. \& Harrison, D. J. Lithographic printing of ceramics. J. Eur. Ceram. Soc. 22, 1-13 (2002).

2 Lin, J. C. \& Wang, C. Y. Effects of surfactant treatment of silver powder on the rheology of its thick-film paste. Mater. Chem. Phys. 45, 136-144 (1996).

3 Goto, T. \& Otsubo, Y. Rheology control of ceramic slurries for sintering of thin films. Nihon Reoroji Gakkaishi (J. Soc. Rheol. Jpn) 29, 205-210 (2001) (in Japanese).

4 Ray, S. S., Yamada, K., Okamoto, M., Fujimoto, Y., Ogami, A. \& Ueda, K. New polylactide/layered silicate nanocomposites. 5. Designing of materials with desired properties. Polymer 44, 6633-6646 (2003).

5 Todoroki, A., Samejima, Y., Hirano, Y. \& Matsuzaki, R. Piezoresistivity of unidirectional carbon/epoxy composites for multiaxial loading. Compos. Sci. Technol. 69, 1841-1846 (2009).

6 Pitarresi, G. \& Galietti, U. A quantitative analysis of the thermoelastic effect in CFRP composite materials. Strain 46, 446-459 (2010).
7 Koyanagi, J., Yoneyama, S., Nemoto, A. \& Melo, J. D. D. Time and temperature dependence of carbon/epoxy interface strength. Compos. Sci. Technol. 70, 13951400 (2010).

8 Futaba, D. N., Hata, K., Yamada, T., Mizuno, K., Yumura, M. \& Iijima, S. Kinetics of water-assisted single-walled carbon nanotube synthesis revealed by a time-evolution analysis. Phys. Rev. Lett. 95, 056104 (2005).

9 Hata, K., Futaba, D. N., Mizuno, K., Namai, T., Yumura, M. \& lijima, S. Water-assisted highly efficient synthesis of impurity-free single-walled carbon nanotubes. Science 306, 1362-1364 (2004).

10 Son, S. Y., Lee, D. H., Kim, S. D., Sung, S. W., Park, Y. S. \& Han, J. H. Synthesis of multi-walled carbon nanotube in a gas-solid fluidized bed. Korean J. Chem. Eng. 23, 838-841 (2006).

11 Wang, Z., Wu, Q., Zhang, F.- Y. \& Cui, Y.- Y. Synthesis of multi-walled carbon nanotube bundles with uniform diameter. Mater. Lett. 61, 1955-1958 (2007).

12 Choi, Y. K., Sugimoto, K. I., Song, S. M. \& Endo, M. Mechanical and thermal properties of vapor-grown carbon nanofiber and polycarbonate composite sheets. Mater. Lett. 59, 3514-3520 (2005)

13 Takahashi, T., Yonetake, K., Koyama, K. \& Kikuchi, T. Polycarbonate crystallization by vapor-grown carbon fiber with and without magnetic field. Macromol. Rapid Commun. 24, 763-767 (2003).

14 Endo, M., Kim, Y. A., Hayashi, T., Nishimura, K., Matusita, T., Miyashita, K. \& Dresselhaus, M. S. Vapor-grown carbon fibers (VGCFs): basic properties and their battery applications. Carbon 39, 1287-1297 (2001).

15 Chung, D. D. L. Comparison of submicron-diameter carbon filaments and conventional carbon fibers as fillers in composite materials. Carbon 39, 1119-1125 (2001).

16 Choi, Y. K., Sugimoto, K. I., Song, S. M. \& Endo, M. Production and characterization of polycarbonate composite sheets reinforced with vapor grown carbon fiber. Compos. $P t$ A-Appl. Sci. Manuf. 37, 1944-1951 (2006).

17 Higgins, B. A. \& Brittain, W. J. Polycarbonate carbon nanofiber composites. Eur. Polym. J. 41, 889-893 (2005).

18 Koyama, T., Tanoue, S. \& lemoto, Y. Preparation and properties of polypropylene/vapor grown carbon fiber composite monofilaments by melt compounding. J. Text. Eng. 55, 73-78 (2009).

19 Koyama, T., Tanoue, S. \& lemoto, Y. Effect if processing conditions on dispersion of vapor grown carbon fiber in a polyamide 6 and the crystalline structure of their composites by melt compounding. Int. Polym. Process 25, 181-187 (2010).

20 Takase, H., Mikata, Y., Matsuda, S. \& Murakami, A. Dispersion of carbon-nanotubes in a polymer matrix by a twin screw extruder. SEIKEI KAKOU 14, 126-131 (2002) (in Japanese).

21 Takase, H., Furukawa, M., Kishi, H. \& Murakami, A. Dispersion of carbon-nanotubes in a polymer matrix by a twin-screw extruder II. SEIKEI KAKOU 17, 50-54 (2005) (in Japanese).

22 Pötschke, P., Fornes, T. D. \& Paul, D. R. Rheological behavior of multiwalled carbon nanotube/polycarbonate composites. Polymer 43, 3247-3255 (2002).

23 Kinloch, I. A., Roberts, S. A. \& Windle, A. H. A rheological study of concentrated aqueous nanotube dispersions. Polymer 43, 7483-7491 (2002).

24 Mason, S. G. Some factors involved in the flocculation of pulp suspensions and the formation of paper. Pulp Paper Mag. Can. 51, 94-98 (1950).

25 Kurath, S. F. The network and viscoelastic properties of wet pulp (I): dynamic mechanical properties. Tappi 42, 953-959 (1959).

26 Thalén, N. \& Wahren, D. Shear modulus and ultimate shear strength of some paper pulp fibre networks. Svensk Papperstidn. 67, 259-264 (1964).

27 Damani, R., Powell, R. L. \& Hagen, N. Viscoelastic characterization of medium consistency pulp suspensions. Can. J. Chem. Eng. 71, 676-684 (1993).

28 Tatsumi, D., Ishioka, S. \& Matsumoto, T. Effect of particle and salt concentrations on the rheological properties of cellulose fibrous suspensions. Nihon Reoroji Gakkaishi (J. Soc. Rheol. Jpn) 27, 243-248 (1999).

29 Tatsumi, D. \& Ishioka, S. Matsumoto, effect of fiber concentration and axial ratio on the rheological properties of cellulose fiber suspensions. Nihon Reoroji Gakkaishi (J. Soc. Rheol. Jpn) 30, 27-32 (2002).

30 Tatsumi, D. Rheology of cellulose fiber disperse systems and cellulose solutions. Nihon Reoroji Gakkaishi (J. Soc. Rheol. Jpn) 35, 251-256 (2007) (in Japanese).

31 de Gennes, P. G. Scaling Concepts in Polymer Physics (Cornell University Press, New York, 1979).

32 Hong, J., Lee, J., Jung, D. \& Shim, S. E. Thermal and electrical conduction behavior of alumina and multiwalled carbon nanotube incorporated poly(dimethyl siloxane). Thermochimica Acta. 512, 34-39 (2010).

33 Sato, E., Takahashi, T., Natsume, T. \& Koyama, K. Development of dispersion and length-evaluation methods of vapor-grown carbon fiber. TANSO 209, 159-164 (2003) (in Japanese).

34 Sato, E., Takahashi, T. \& Koyama, K. Comparison of the length of vapor-grown carbon fiber before and after mixing process. Kobunshi Ronbunshu. 61, 144-148 (2004) (in Japanese).

35 Nithikarnjanatharn, J., Ueda, H., Tanoue, S., Uematsu, H. \& lemoto, Y. Properties of poly(carbonate)/vapor-grown carbon fiber composite prepared by melt compounding. J. Text. Eng. 57, 97-106 (2011). 